\title{
Association Between Household Size, Residential Area, and Osteoporosis: Analysis of 2008-2011 KNHANES
}

Sung-Woo Kim¹, Kwi-Hyun Bae1, Jae-Han Jeon'1, Yeon-Kyung Choi', Mi-Kyung Kim², Hye-Soon Kim², Hyun-Ae Seo ${ }^{3}$, Eui Hyun Kim³, Keun-Gyu Park ${ }^{1}$

) Division of Endocrinology and Metabolism, Department of Internal Medicine, Kyungpook National University School of Medicine, Daegu, South Korea

2) Division of Endocrinology and Metabolism, Department of Internal Medicine, Keimyung University School of Medicine, Daegu, South Korea

Division of Endocrinology and Metabolism, Department of Internal Medicine, Daegu Fatima Hospital, Daegu, South Korea

\section{Introdituetion and objectites}

- The prevalence of single-person households has rapidly increased in Korea. Individuals living alone and in rural areas may have a higher risk of various metabolic diseases due to differences in lifestyle. However, few studies have investigated the association of household size and residential area with healthrelated problems. This study aimed to evaluate the association of household size and residential area with risk of osteoporosis in postmenopausal women.

\section{Bosmlts}

\section{Baseline characteristics of participants}

\begin{tabular}{|c|c|c|c|}
\hline & \multicolumn{2}{|c|}{ Postmenopausal women $(n=3058)$} & \multirow[b]{2}{*}{$P$ value } \\
\hline & $\begin{array}{l}\text { Rural residents } \\
(\mathrm{n}=969)\end{array}$ & $\begin{array}{l}\text { Urban residents } \\
(\mathrm{n}=2089)\end{array}$ & \\
\hline Age, y & $65.2 \pm 0.3$ & $62.4 \pm 0.2$ & $<0.001$ \\
\hline $\mathrm{BMI}, \mathrm{kg} / \mathrm{m}^{2}$ & $24.1 \pm 0.1$ & $24.2 \pm 0.1$ & 0.233 \\
\hline Systolic BP, mmHg & $129.7 \pm 0.6$ & $127.7 \pm 0.4$ & 0.002 \\
\hline Diastolic BP, mmHg & $78.2 \pm 0.3$ & $78.2 \pm 0.2$ & 0.926 \\
\hline Fasting glucose, $\mathrm{mg} / \mathrm{dL}$ & $99.7 \pm 0.7$ & $101.1 \pm 0.5$ & 0.269 \\
\hline Total cholesterol, mg/dL & $200.1 \pm 1.2$ & $202.0 \pm 0.8$ & 0.313 \\
\hline Vitamin $\mathrm{D}, \mathrm{ng} / \mathrm{mL}$ & $19.8 \pm 0.2$ & $17.7 \pm 0.2$ & $<0.001$ \\
\hline PTH, pg/mL & $70.7 \pm 1.1$ & $68.6 \pm 0.7$ & 0.133 \\
\hline Age at menopause, $y$ & $48.5 \pm 0.2$ & $49.7 \pm 0.1$ & $<0.001$ \\
\hline Number of pregnancies, $n$ & $5.2 \pm 0.1$ & $4.8 \pm 0.1$ & $<0.001$ \\
\hline Food intake, g/day & $1023.9 \pm 16.4$ & $1153.2 \pm 14.2$ & $<0.001$ \\
\hline \multicolumn{4}{|l|}{ Nutrient support } \\
\hline Total energy intake, kcal/day & $1554.1 \pm 18.2$ & $1568.2 \pm 13.8$ & 0.630 \\
\hline Protein intake, g/day & $50.3 \pm 0.9$ & $53.3 \pm 0.6$ & 0.005 \\
\hline Fat intake, g/day & $19.9 \pm 0.5$ & $24.2 \pm 0.4$ & $<0.001$ \\
\hline Calcium intake, $\mathrm{mg} / \mathrm{day}$ & $400.4 \pm 9.7$ & $441.5 \pm 9.3$ & 0.012 \\
\hline Working hours, h/week & $27.7 \pm 0.8$ & $15.2 \pm 0.5$ & $<0.001$ \\
\hline Physical activity (high/moderate/walk), & $109 / 137 / 245$ & $241 / 180 / 634$ & \\
\hline $\mathrm{n}, \%$ & $(11.2 \% / 14.1 \% / 25.2 \%)$ & $(11.5 \% / 8.6 \% / 30.3 .0 \%)$ & 0.202 \\
\hline Heavy alcoholics, n, \% & $12(1.2 \%)$ & $23(1.1 \%)$ & 0.740 \\
\hline Cigarette smoking (current/ex), n, \% & $22 / 43(2.3 \% / 4.4 \%)$ & $93 / 76(4.5 \% / 3.6 \%)$ & 0.029 \\
\hline DM, n, \% & $108(11.1 \%)$ & $278(13.3 \%)$ & 0.094 \\
\hline HTN, n, \% & $406(41.9 \%)$ & $796(38.1 \%)$ & 0.046 \\
\hline Anti-hypertensive drug use, $n, \%$ & $355(36.6 \%)$ & $686(32.8 \%)$ & 0.103 \\
\hline Thyroid disease, n, \% & $53(5.5 \%)$ & $179(8.6 \%)$ & 0.003 \\
\hline History of cancer, n, \% & $40(4.1 \%)$ & $85(4.1 \%)$ & 0.916 \\
\hline Number of people per households, $n$ & $2.4 \pm 0.0$ & $2.8 \pm 0.0$ & $<0.001$ \\
\hline Single-person households, n, \% & $188(19.4 \%)$ & $276(13.2 \%)$ & $<0.001$ \\
\hline Osteoporosis, n, \% & $406(41.9 \%)$ & $646(30.9 \%)$ & $<0.001$ \\
\hline Vertebral fracture, n, \% & $17(1.8 \%)$ & $20(1.0 \%)$ & 0.091 \\
\hline
\end{tabular}

All data are expressed as unweighted mean \pm standard error or number (\%). All $P$ values were obtained using the Student's t-test or Chi-square test. Abbreviations: BMI, body mass index; BP, blood pressure; PTH, parathyroid hormone; DM, diabetes melitus; HTN, hypertension

3. BMD according to household size and residential area

\begin{tabular}{lllll}
\hline \multicolumn{4}{c}{ Rural area } & \multicolumn{3}{l}{ Urban area } \\
\cline { 2 - 5 } & \multicolumn{1}{l}{ Single } & Two-more & Single & Two-more (Ref) \\
\hline Lumbar spine BMD & & & \\
Unadjusted & $0.734 \pm 0.012^{* *}$ & $0.800 \pm 0.007 *$ & $0.778 \pm 0.007 * *$ & $0.821 \pm 0.004$ \\
Adjusted & $0.786 \pm 0.016^{* *}$ & $0.807 \pm 0.015$ & $0.819 \pm 0.015$ & $0.816 \pm 0.014$ \\
Total femur BMD & & & & \\
Unadjusted & $0.716 \pm 0.010^{* *}$ & $0.783 \pm 0.006$ & $0.741 \pm 0.006 * *$ & $0.788 \pm 0.003$ \\
Adjusted & $0.754 \pm 0.012$ & $0.769 \pm 0.011$ & $0.769 \pm 0.010$ & $0.761 \pm 0.010$ \\
Femoral neck BMD & & & & \\
Unadjusted & $0.574 \pm 0.009 * *$ & $0.634 \pm 0.005$ & $0.586 \pm 0.005 * *$ & $0.639 \pm 0.003$ \\
Adjusted & $0.619 \pm 0.011$ & $0.630 \pm 0.010$ & $0.624 \pm 0.010$ & $0.624 \pm 0.009$ \\
\hline
\end{tabular}

${ }^{*} P<0.05$ (statistical significance). ${ }^{* *} P<0.01$ (statistical significance)

\section{Wothoc}

This cross-sectional study enrolled 3058 postmenopausal women from the 2008-2011 Korea National Health and Nutrition Examination Survey (KNHANES). We examined the association between bone mineral density (BMD) and household size and residential area

2. General characteristics of participants stratified by household size and residential area

\begin{tabular}{|c|c|c|c|c|}
\hline & \multicolumn{2}{|l|}{ Rural } & \multicolumn{2}{|l|}{ Urban } \\
\hline & $\begin{array}{l}\text { Single } \\
(n=194)\end{array}$ & $\begin{array}{l}\text { Two-more } \\
(\mathrm{n}=775)\end{array}$ & $\begin{array}{l}\text { Single } \\
(n=297)\end{array}$ & $\begin{array}{l}\text { Two-more } \\
(\mathrm{n}=1792) \text { (Ref) }\end{array}$ \\
\hline Age, y & $69.8 \pm 0.6$ ** & $64.0 \pm 0.3^{* *}$ & $68.2 \pm 0.5^{* *}$ & $61.5 \pm 0.2$ \\
\hline BMI, kg/m² & $23.8 \pm 0.3$ & $24.2 \pm 0.1$ & $24.6 \pm 0.2$ * & $24.2 \pm 0.1$ \\
\hline Systolic BP, mmHg & $131.4 \pm 1.2^{* *}$ & $129.3 \pm 0.6^{* *}$ & $132.0 \pm 1.1^{* *}$ & $127.0 \pm 0.4$ \\
\hline Diastolic BP, mmHg & $78.5 \pm 0.8$ & $78.1 \pm 0.4$ & $78.4 \pm 0.6$ & $78.2 \pm 0.2$ \\
\hline Fasting glucose, $\mathrm{mg} / \mathrm{dL}$ & $100.2 \pm 1.5$ & $99.5 \pm 0.8$ & $101.8 \pm 1.2$ & $100.9 \pm 0.6$ \\
\hline Total cholesterol, mg/dL & $199.5 \pm 2.6$ & $200.3 \pm 1.3$ & $200.8 \pm 2.2$ & $202.2 \pm 0.9$ \\
\hline Vitamin $\mathrm{D}, \mathrm{ng} / \mathrm{mL}$ & $20.8 \pm 0.5^{* *}$ & $19.5 \pm 0.2^{* *}$ & $18.4 \pm 0.4$ & $17.6 \pm 0.2$ \\
\hline PTH, pg/mL & $74.4 \pm 2.6$ * & $69.7 \pm 1.2$ & $71.7 \pm 1.7$ & $68.1 \pm 0.8$ \\
\hline Age at menopause, $y$ & $48.0 \pm 0.37^{* *}$ & $48.7 \pm 0.17^{* *}$ & $49.0 \pm 0.31$ ** & $49.8 \pm 0.10$ \\
\hline Number of pregnancies, $\mathrm{n}$ & $5.7 \pm 0.17^{* *}$ & $5.1 \pm 0.08^{* *}$ & $5.4 \pm 0.18^{* *}$ & $4.7 \pm 0.06$ \\
\hline Food intake, g/day & $900.9 \pm 31.6 * *$ & $1055.0 \pm 18.7^{* *}$ & $976.0 \pm 35.2$ ** & $1182.6 \pm 15.4$ \\
\hline \multicolumn{5}{|l|}{ Nutrient support } \\
\hline Total energy intake, kcal/day & $1438.4 \pm 34.8^{* *}$ & $1583.3 \pm 20.9$ & $1457.4 \pm 34.7^{* *}$ & $1586.6 \pm 15.0$ \\
\hline Protein intake, g/day & $44.2 \pm 1.5^{* *}$ & $51.8 \pm 1.0$ & $47.7 \pm 1.5^{\star *}$ & $54.2 \pm 0.7$ \\
\hline Fat intake, g/day & $16.1 \pm 0.9$ ** & $20.9 \pm 0.6$ * & $20.1 \pm 1.1^{\star *}$ & $24.9 \pm 0.4$ \\
\hline Calcium intake, $\mathrm{mg} / \mathrm{day}$ & $377.6 \pm 25.6$ * & $406.1 \pm 10.3$ * & $410.2 \pm 20.0$ & $446.7 \pm 10.3$ \\
\hline Working hours, h/week & $18.0 \pm 1.6$ & $30.1 \pm 0.9$ ** & $9.8 \pm 1.1^{* *}$ & $16.1 \pm 0.6$ \\
\hline $\begin{array}{l}\text { Physical activity } \\
\text { (high/moderate/walk), } \mathrm{n}\end{array}$ & $22 / 26 / 53$ & $87 / 111 / 192$ & 28/28/90 & $213 / 152 / 544$ \\
\hline Heavy alcoholics, n, \% & $5(2.6 \%)$ & $7(0.9 \%)$ & $5(1.7 \%)$ & $18(1.0 \%)$ \\
\hline $\begin{array}{l}\text { Cigarette smoking } \\
\text { (current/ex), n, \% }\end{array}$ & $\begin{array}{l}14 / 4 \\
(7.2 \% / 2.1 \%)\end{array}$ & $\begin{array}{l}29 / 18 \\
(3.7 \% / 2.3 \%)\end{array}$ & $\begin{array}{l}18 / 23^{* *} \\
(6.1 \% / 7.7 \%)\end{array}$ & $\begin{array}{l}58 / 70 \\
(3.2 \% / 3.9 \%)\end{array}$ \\
\hline $\mathrm{DM}, \mathrm{n}, \%$ & $23(11.9 \%)$ & $85(11.0 \%)$ & $47(15.8 \%)$ & $231(12.9 \%)$ \\
\hline HTN, n, \% & $87(44.8 \%)^{*}$ & $319(41.2 \%)^{*}$ & $148(49.8 \%)^{* *}$ & $648(36.2 \%)$ \\
\hline Anti-hypertensive drug use, $\mathrm{n}, \%$ & $80(44.2 \%)^{*}$ & $280(36.1 \%)$ & $133(44.8 \%)^{* *}$ & $597(33.3 \%)$ \\
\hline Thyroid disease, n, \% & $6(3.1 \%)^{* *}$ & $47(6.1 \%)^{*}$ & $21(7.1 \%)$ & $158(8.8 \%)$ \\
\hline History of cancer, n, \% & $10(5.2 \%)$ & $36(4.6 \%)$ & $16(5.4 \%)$ & $85(4.7 \%)$ \\
\hline Osteoporosis, n, \% & $114(58.8 \%)^{* *}$ & $307(39.6 \%)^{* *}$ & $130(43.8 \%)^{* *}$ & $555(31.0 \%)$ \\
\hline Vertebral fracture, n, \% & $4(2.1 \%)$ & $14(1.8 \%)$ & $6(2.0 \%)$ & $17(0.9 \%)$ \\
\hline
\end{tabular}

4. Osteoporosis and fracture risk according to household size and residential area

\begin{tabular}{|c|c|c|c|c|}
\hline & \multicolumn{2}{|l|}{ Rural area } & \multicolumn{2}{|l|}{ Urban area } \\
\hline & Single & Two-more & Single & Two-more \\
\hline \multicolumn{5}{|c|}{ Osteoporosis of lumbar spine } \\
\hline Unadjusted & $\begin{array}{l}3.155^{* *} \\
(2.143-4.646)\end{array}$ & $\begin{array}{l}1.383^{*} \\
(1.064-1.797)\end{array}$ & $\begin{array}{l}1.633^{* *} \\
(1.194-2.233)\end{array}$ & 1 (Ref) \\
\hline Model 1 & $\begin{array}{l}1.622 \text { * } \\
(1.045-2.517)\end{array}$ & $\begin{array}{l}1.152 \\
(0.874-1.517)\end{array}$ & $\begin{array}{l}0.853 \\
(0.592-1.229)\end{array}$ & 1 (Ref) \\
\hline Model 2 & $\begin{array}{l}1.667^{*} \\
(1.083-2.565)\end{array}$ & $\begin{array}{l}1.137 \\
(0.861-1.502)\end{array}$ & $\begin{array}{l}0.879 \\
(0.610-1.266)\end{array}$ & 1 (Ref) \\
\hline \multicolumn{5}{|c|}{ Vertebral fracture } \\
\hline Unadjusted & $\begin{array}{l}3.518 \\
(0.880-14.054)\end{array}$ & $\begin{array}{l}2.617^{*} \\
(1.099-6.228)\end{array}$ & $\begin{array}{l}2.675 \\
(0.976-7.336)\end{array}$ & 1 (Ref) \\
\hline Model 1 & $\begin{array}{l}1.884 \\
(0.362-9.813)\end{array}$ & $\begin{array}{l}1.848 \\
(0.756-4.515)\end{array}$ & $\begin{array}{l}1.265 \\
(0.444-3.608)\end{array}$ & 1 (Ref) \\
\hline Model 2 & $\begin{array}{l}1.963 \\
(0.347-11.107)\end{array}$ & $\begin{array}{l}1.865 \\
(0.783-4.442)\end{array}$ & $\begin{array}{l}1.309 \\
(0.447-3.839)\end{array}$ & 1 (Ref) \\
\hline
\end{tabular}

Model 1: adjusted for age, body mass index, number of pregnancies, duration of menopause, systolic blood pressure, parathyroid hormone, vitamin $\mathrm{D}$ levels, hypertension, and thyroid disease.

Model 2: adjusted for age, body mass index, number of pregnancies, duration of menopause, systolic blood pressure, parathyroid hormone, vitamin D levels, hypertension, thyroid disease, working hours, physical activity, heavy alcoholics, smoking status, daily food intake, and nutrient support.

Data were analyzed by the complex samples logistic regression model.

All data are expressed as odds ratio (95\% confidence interval).

${ }^{*} P<0.05$ (statistical significance). ${ }^{* *} P<0.01$ (statistical significance)

\section{Bonclnsions}

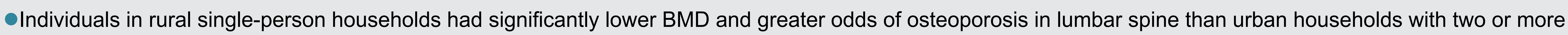
individuals. The results of this study suggest that individuals living in rural single-person households may benefit from more careful screening for osteoporosis. 